\title{
Quel lien entre les pratiques sportives des élèves et leur performance et leur bien-être ?
}

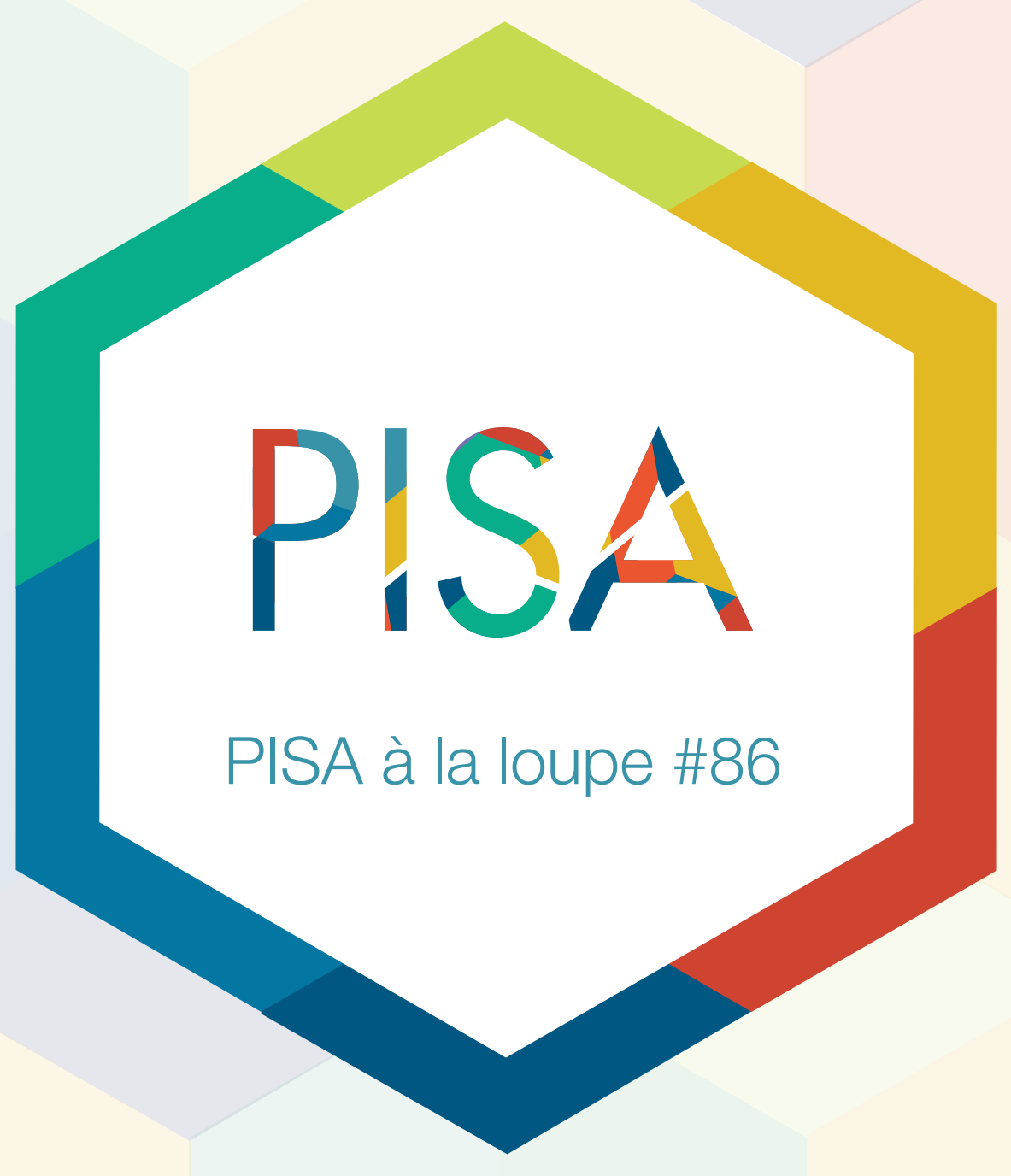




\section{Quel lien entre les pratiques sportives des élèves et leur performance et leur bien-être ?}

- Dans les pays de l'OCDE, 52 \% des élèves indiquent pratiquer une activité physique intense (qui les fait transpirer et entraîne un essoufflement) au moins trois jours par semaine ; en moyenne, les garçons pratiquent ce type d'activité un jour de plus par semaine que les filles.

- II existe une relation positive entre la fréquence de la pratique d'une activité physique intense et le bien-être des élèves.

- Les élèves pratiquant une activité physique modérée au moins un jour par semaine tendent à obtenir de meilleurs résultats aux évaluations PISA que ceux n'en pratiquant aucune. En revanche, les élèves pratiquant une activité physique intense chaque jour de la semaine obtiennent un score plus faible en sciences que ceux faisant de l'exercice entre un et six jours par semaine.

Le sport occupe une place essentielle dans la vie des élèves. La pratique régulière d'une activité physique peut réduire les risques d'obésité, les troubles anxieux, le manque d'estime de soi et le harcèlement chez les adolescents, et les aider à mener une vie plus active et plus saine une fois à l'âge adulte. Toutefois, les cours d'éducation physique et la pratique d'activités sportives en dehors du cadre scolaire sont en concurrence avec nombre d'autres activités essentielles dans l'emploi du temps déjà chargé des élèves, notamment les devoirs et l'étude. Les professionnels de l'éducation et les parents peuvent se demander si les enfants consacrent assez (ou peut-être trop) de temps à la pratique d'activités physiques, et dans quelle mesure elle est liée aux résultats scolaires des élèves et à leur bien-être.

\section{La plupart des élèves pratiquent une activité physique régulière.}

En 2015, l'enquête PISA a interrogé les élèves de 15 ans sur leur pratique d'une activité physique intense (activité d'une durée d'au moins 20 minutes par jour les faisant transpirer et entraînant un essoufflement, telle que la course à pied, le tennis ou le football). II en ressort que la plupart des élèves pratiquent une activité physique régulière. En moyenne, dans les pays de l'OCDE, $52 \%$ des élèves pratiquent au minimum 3 jours par semaine une activité d'une durée d'au moins 20 minutes les faisant transpirer et entraînant un essoufflement. Au Danemark, aux États-Unis, en Islande, en Norvège, en Pologne et en Suède, plus de $60 \%$ des élèves sont dans ce cas, contre moins de $40 \%$ au Brésil, en Colombie, en Corée, au Costa Rica, aux Émirats arabes unis, en France, à Hong-Kong (Chine), à Macao (Chine), à Singapour, en Tunisie et en Turquie.

Dans la plupart des pays et économies participant à l'enquête PISA, les garçons tendent à pratiquer plus souvent une activité physique intense que les filles : ils indiquent ainsi le faire 4.4 jours par semaine, en moyenne, contre seulement 3.5 jours pour les filles. Les différences entre les sexes sont les plus faibles dans les pays d'Europe du Nord et de l'Est, mais tendent à être marquées en Corée, au Japon et dans les pays d'Amérique latine.

\section{La pratique régulière d'une activité physique est liée à un niveau plus élevé de bien-être chez les élèves.}

Une nette association positive s'observe entre la pratique d'une activité physique et le bien-être des élèves. D'après l'enquête PISA, les élèves pratiquant une activité physique modérée (activité d'une durée d'au moins 60 minutes par jour augmentant le rythme cardiaque et faisant transpirer, comme marcher, monter les escaliers, aller à l'école à vélo) tendent à faire part d'un niveau plus élevé de bien-être psychosocial que ceux ne pratiquant aucune activité physique. Les élèves pratiquant une activité physique modérée plus souvent durant la semaine sont ainsi plus susceptibles de valoriser le travail en équipe et la coopération. En outre, dans la plupart des pays, les élèves faisant de l'exercice au moins trois jours par semaine font part d'une plus grande satisfaction à l'égard de la vie que ceux ne pratiquant aucune activité physique en dehors de l'école. Les élèves ne pratiquant aucune activité physique modérée évaluent leur satisfaction à l'égard de la vie à un niveau de 6.9 sur une échelle allant de 0 (pire vie possible) à 10 (meilleure vie possible), contre 7.2 pour ceux faisant de l'exercice une ou deux fois par semaine, et 7.5 pour ceux pratiquant une activité physique intense au moins 3 jours par semaine. La prudence est toutefois de mise lors de l'interprétation de cette relation, car les élèves indiquant ne pratiquer aucune activité physique peuvent souffrir de handicaps physiques qui les en empêchent.

Les élèves ne pratiquant aucune forme d'activité physique en dehors de l'école - ni intense, comme la course à pied, ni modérée, comme la marche ou la danse - tendent à se situer au bas de l'échelle sur différents indicateurs psychologiques et sociaux, et sont plus susceptibles d'adopter des comportements à risque. En moyenne, dans les pays de l'OCDE, les élèves indiquant pratiquer une activité physique modérée ou intense sont moins susceptibles que ceux ne pratiquant aucune forme d'activité physique en dehors de l'école : de se dire angoissés par le travail scolaire (différence de 3 points de pourcentage) ; de se sentir comme des étrangers à l'école (différence de 7 points de pourcentage) ; de sécher les cours (différence de 3 points de pourcentage) ; et d'être souvent harcelés (différence de 2 points de pourcentage).

\section{Toutefois, la pratique plus fréquente d'une activité physique ne rime pas nécessairement avec l'obtention de meilleurs résultats d'apprentissage.}

Certains travaux de recherche semblent indiquer que la pratique régulière d'une activité sportive peut améliorer la mémoire, la persévérance et les capacités d'autorégulation chez les adolescents. En moyenne, dans les pays de l'OCDE, la pratique d'une activité physique modérée d'une durée 
Pourcentage d'élèves pratiquant une activité physique intense au moins trois jours par semaine

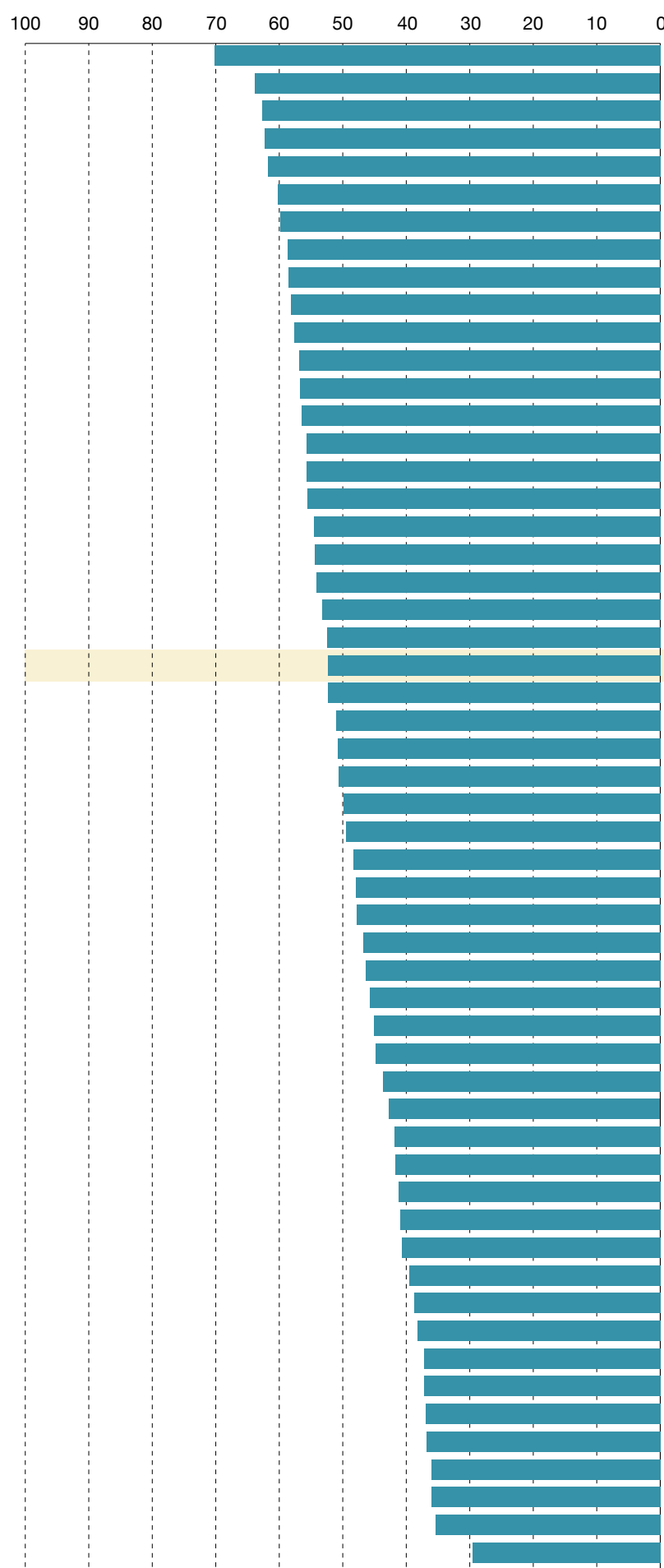

Nombre moyen de jours de pratique d'une activité physique intense, selon le sexe des élèves

Filles Garçons Journées

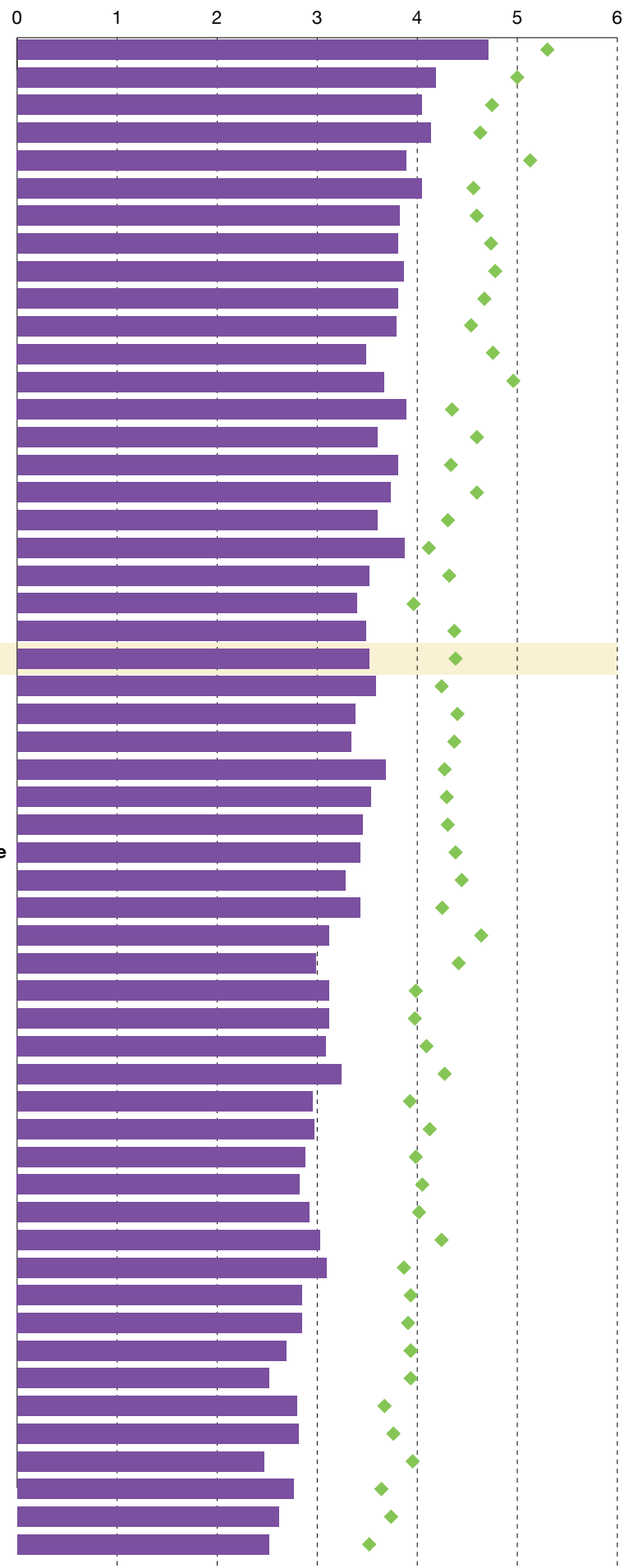

*L'acronyme P-S-J-G (Chine) désigne les quatre municipalités/provinces chinoises participant à l'enquête PISA : Pékin, Shanghai, Jiangsu et Guangdong. 'La Lituanie est devenue membre de l'OCDE le 5 juillet 2018 et n'est pas incluse dans la moyenne OCDE.

Les pays et économies sont classés par ordre décroissant du pourcentage d'élèves pratiquant une activité physique intense au moins trois jours par semaine. Source : OCDE, Base de données PISA 2015, tableaux III.11.9 et III.11.13. 
Satisfaction des élèves à l'égard de la vie, selon le nombre de jours de pratique d'une activité physique par semaine

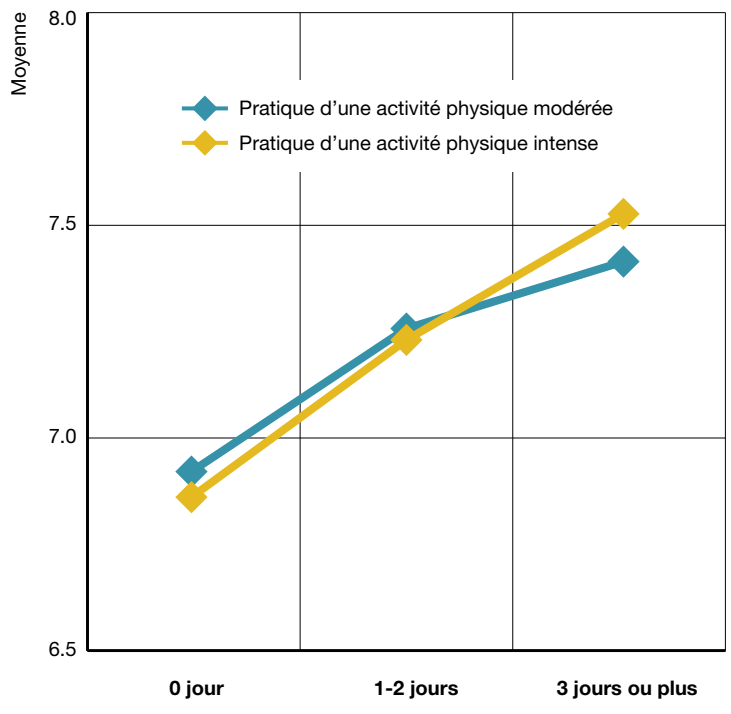

Pourcentage d'élèves faisant part des retombées suivantes

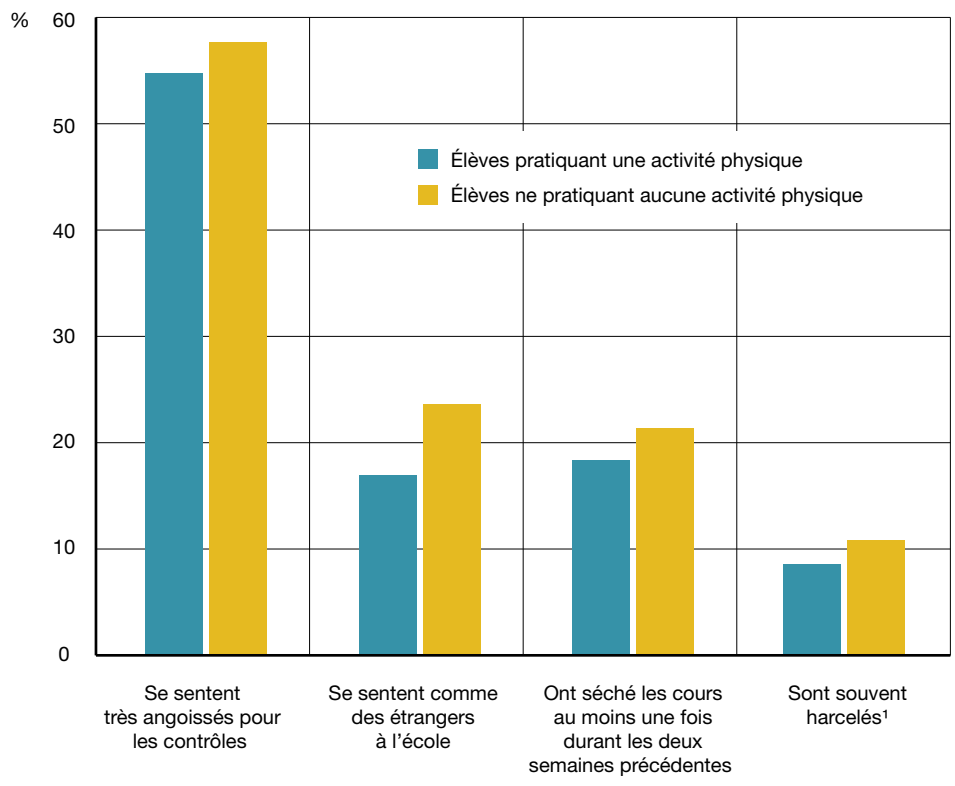

1Par élèves souvent harcelés, on entend ceux se situant dans le décile supérieur de l'indice d'exposition au harcèlement, tous pays et économies confondus.

Remarque : Toutes les différences sont statistiquement significatives entre 3 jours ou plus d'activité physique par semaine et 0 jour, et entre les élèves pratiquant une activité physique et ceux n'en pratiquant aucune.

Source : OCDE, Base de données PISA 2015, tableaux III.11.16 et III.11.18.

d'au moins 60 minutes par jour semble liée, bien que faiblement, à l'obtention de meilleurs résultats scolaires. Dans la plupart des pays et économies participant à l'enquête PISA, il existe une relation faible, mais positive, entre le nombre de jours de pratique d'une activité physique modérée en dehors de l'école et la performance moyenne des élèves en sciences. De même, une relation limitée, mais positive, s'observe entre la pratique d'une activité physique modérée et la capacité des élèves à résoudre des problèmes dans un cadre collaboratif.

Cependant, la relation inverse s'observe avec la pratique d'une activité physique intense. Les élèves pratiquant ce type d'activité en dehors de l'école sept jours par semaine obtiennent de moins bons scores en sciences que leurs pairs. Un jour supplémentaire de pratique d'une activité physique intense est associé à une baisse de 3 points du score en sciences, en moyenne, dans les pays de l'OCDE. Les élèves pratiquant une activité physique intense chaque jour obtiennent 25 points de moins en sciences que leurs pairs pratiquant ce type d'activité quatre jours par semaine. Dans certains des pays/économies les plus performants à l'évaluation PISA de sciences, tels que la Corée, Hong-Kong (Chine) et Singapour, la relation négative entre un jour supplémentaire de pratique d'une activité physique intense et la performance en sciences est plus forte que celle observée dans les autres pays.

Ces tendances n'indiquent pas nécessairement l'existence d'un lien de causalité entre la pratique d'une activité physique et la performance scolaire des élèves. Ainsi, les élèves scolarisés dans des établissements très compétitifs peuvent limiter le nombre d'heures qu'ils dédient à la pratique d'une activité physique, compte tenu du temps qu'ils doivent consacrer à leurs devoirs et à la préparation de leurs cours. Les élèves pratiquant un grand nombre d'activités sportives appartiennent peut-être à une catégorie d'élèves accordant une plus grande priorité à la réussite sportive qu'aux résultats scolaires.

\section{Pour conclure}

La pratique d'une activité sportive n'est pas nécessairement liée à l'obtention de meilleurs résultats dans les disciplines académiques, mais est fortement associée au bien-être des adolescents et des adultes qu'ils deviendront. Les professionnels de l'éducation et l'école peuvent donc améliorer le bien-être des élèves en leur proposant des cours d'éducation physique de qualité et en les aidant à maintenir une activité physique régulière, à l'école comme ailleurs. 
Score moyen des élèves en sciences, selon le nombre de jours de pratique d'une activité physique modérée par semaine

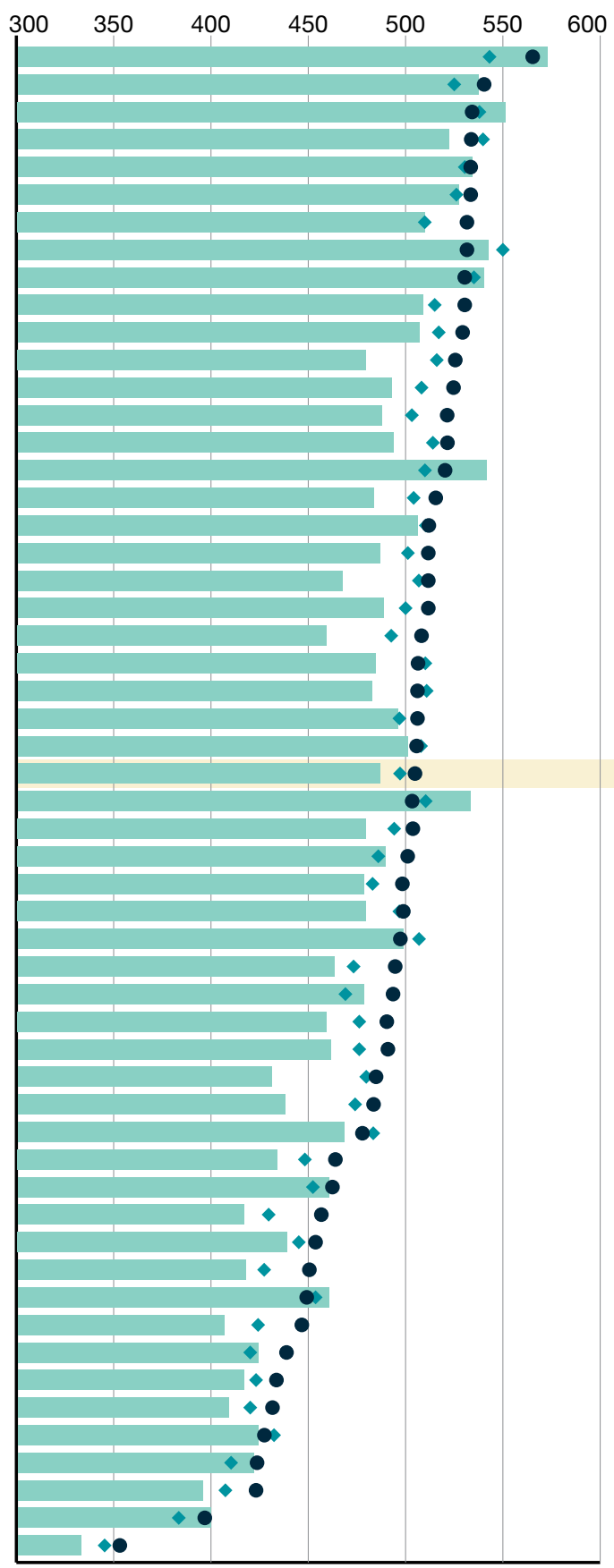

Évolution du score en sciences associée à un jour supplémentaire de... $\begin{array}{lr}\text { Pratique d'une } & \begin{array}{r}\text { Pratique d'une } \\ \text { activité physique } \\ \text { modérée }\end{array} \\ \text { activité physique } \\ \text { intense }\end{array}$

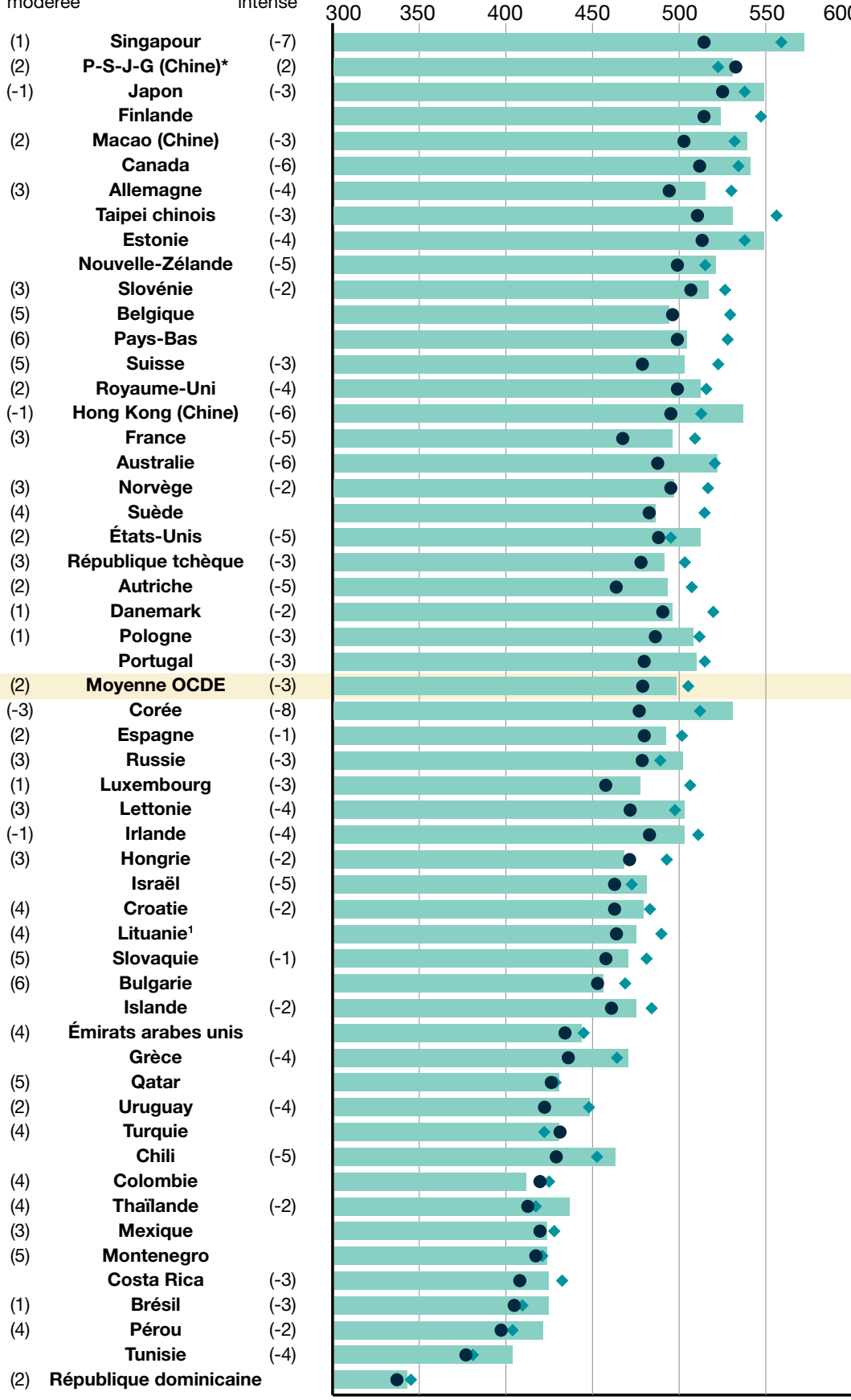

*L'acronyme P-S-J-G (Chine) désigne les quatre municipalités/provinces chinoises participant à l'enquête PISA : Pékin, Shanghai, Jiangsu et Guangdong

'La Lituanie est devenue membre de l'OCDE le 5 juillet 2018 et n'est pas incluse dans la moyenne OCDE.

Remarque : Les évolutions statistiquement significatives du score en sciences associées à un jour supplémentaire de pratique d'une activité physique modérée ou intense, après contrôle du statut socio-économique et du sexe des élèves, sont indiquées en regard des noms des pays/économies.

Les pays et économies sont classés par ordre décroissant de la pratique d'une activité physique modérée sept jours par semaine.

Source : OCDE, Base de données PISA 2015, tableaux III.11.11a, III.11.12a et III.11.15. 


\section{Pour tout complément d'information}

\section{Contacter : Judit Pál (judit.pal@oecd.org)}

Consulter: Mo, J. (2018), "What kinds of activities are common among teenagers who work well with others? ", PISA à la loupe, n 84, Éditions OCDE, Paris, https://doi.org/10.1787/7cb73373-en.

OCDE (2017), Résultats du PISA 2015 Volume III) : Le bien-être des élèves, PISA, Éditions OCDE, Paris, https://doi.org/10.1787/9789264288850-fr.

Le mois prochain : Les jeunes de 15 ans sont-ils devenus plus « verts »?

Cet ouvrage est publié sous la responsabilité du Secrétaire général de l'OCDE. Les opinions et les interprétations exprimées ne reflètent pas nécessairement les avis des pays membres de l'OCDE.

Ce document, ainsi que les données et cartes qu'il peut comprendre, sont sans préjudice du statut de tout territoire, de la souveraineté s'exerçant sur ce dernier, du tracé des frontières et limites internationales, et du nom de tout territoire, ville ou région.

Les données statistiques concernant Israël sont fournies par et sous la responsabilité des autorités israéliennes compétentes. L'utilisation de ces données par l'OCDE est sans préjudice du statut des hauteurs du Golan, de Jerusalem-Est et des colonies de peuplement israéliennes en Cisjordanie aux termes du droit international.

Ce texte est disponible sous licence Attribution - Pas d'Utilisation Commerciale - Partage dans les Mêmes Conditions 3.0 Organisations Internationales (CC BY-NC-SA 3.0 IGO). Pour toute information spécifique quant à l'étendue et aux termes de la licence ainsi que d'une possible utilisation commercial de ce texte et pour toute usage de données PISA, prière de consulter les Conditions d'utilisation à http://www.oecd.org/fr/conditionsdutilisation. 